\title{
Research on the Spatial Distribution Pattern of Producer Service Firms in Urban Harbin
}

\author{
Yang-yang Zhao *, Ji-ping Liu , Fu-hao Zhang, Meng Liu \\ Research Center of Government GIS, Chinese Academy of Surveying and Mapping, nhyyangyang@126.com, liujp@casm.ac.cn, \\ zhangfh@casm.ac.cn,liuflame123@gmail.com. \\ * Corresponding author
}

\begin{abstract}
The spatial distribution pattern of producer service firms is a significant part of the spatial distribution of industry. In this paper a strategy to get the spatial distribution pattern of producer service firms was proposed by using cartographic generalization, the local Getis-Ord Gi* statistic and overlay method. First, a basic geographic layer, main contained road and residential, should be drawn utilizing cartographic generalization. Second, an enterprise thematic layer, shown the agglomeration effect of producer service firms, was drawn using the local Getis-Ord Gi* statistic. Finally, get the spatial distribution pattern of producer service firms overlaying the basic geographic layer with enterprise thematic layer. Taking urban Harbin, China as a case study, an empirical study was conducted with data of producer service firms in 2016. The results indicated four distribution regions: (i) it showed the high hot agglomeration effect in centre urban Harbin; (ii) it appeared hot agglomeration effect in both north and south of centre urban Harbin; (iii) some of producer service firms randomly distributed in the surrounding area; and (iv) a fraction of producer service firms scattered in the peripheral area.
\end{abstract}

Keywords: Spatial distribution pattern, Spatial autocorrelation, Agglomeration effect, Producer service firms

\section{Introduction}

Producer service firms is one of the service industries from the perspective of service users, in correspondence with the consumer-services (Qun-yi Z., \& Yi-xing Z. 2007). With the development of the economy, Producer service firms increasingly occupy an important place in the national economy and employment. It has become the major part of service industries now. In academia, spatial distribution pattern is a key task for industrial structure in producer-service, because it can help people to learn about the distribution characteristics and make decisions.

Harry I. Greenfield first put forward the concept of producer service firms when he studied the classification of services. that was the externalization and marketization of production services essentially (Greenfield, H. I. 1966). This paper build analysis and interpretation on the foundations provided by literatures documenting the internationalization of producer service firms such as accountancy, advertising, architecture, finance and law (Taylor, P. J. et al.2014; Bagchi-Sen and Sen 1997; Bryson et al. 2004; Daniels 1993; Faulconbridge et al. 2008; Jones 2002).

Scholars started to study the distribution characteristics of the producer service firms in the 1980s and formed different viewpoints. Two kinds of views on the spatial distribution of the producer service firms of inner city were put forward in 1980s. One view is that the spatial centralized characteristics of the producer service firms would become more and more obvious. Because enterprises, to enhance their control ability, the timeliness and validity decision-making, need to carry out the faceto-face exchanges with other enterprises frequently. Another view is that the spatial centralized characteristics of inner city would change because the development of traffic and communication tools would reduce the exchange cost. Enterprises would get a close contact with others even in the suburbs(Illeris S. 1996).In the late of 1990s, scholars began to detect the spatial distribution pattern of specific producer service firms(Stanback 1991, Shearmur R., \& Alvergne C. 2002). The results shown that the two viewpoints above existed at the same time, a part of producer service firms still concentrated highly in the CBD, and another part of firms located in the suburbs. Research on the spatial distribution characteristics of producer service firms were drawn more and more attention of scholars. Yin Chen and Jin-yong $\mathrm{Li}$ put forward that both the agglomeration and diffusion were two basic organization forms of producer service firms' distribution(Yin C., \& Jin-yong L. 2004). Qun-yi Zhao and Yi-xing Zhou, using the simulation of spatial distribution, the spatial autocorrelation and factor analysis, carried out the spatial distribution pattern of producer service firms in Beijing metropolitan area(Qun-yi Z., \& Yi-xing Z. 2007). Yu-fang Shen and Shu-hua Li summarized the layout structure of the producer service firms from three regional levels in the Yangtze River delta. The spatial layout structure of the producer service firms is one core region and two flanks (Yu-fang S., \& Shu-hua L. 2011). In short, scholars have researched the distribution of productive service firms using different methods various from scales. Most of all were 
concentrated on the distribution characteristics, agglomeration effects and distribution pattern. What's more, many existing studies focused on the metropolis, such as Beijing, Shanghai, and seldom focused on the second- and third-class cities. This paper selected Urban Harbin as the study area and proposed a strategy to analyse the spatial distribution pattern that utilized cartographic generalization, the local Getis-Ord Gi* statistic of exploratory data analysis (ESDA) and the overlay method of GIS. Using the organization data which provided the positions of the enterprises, experiment showed that there were four distribution regions of producer service firms in urban Harbin.

\section{Method}

This paper proposed a strategy to analyse the spatial distribution pattern of producer service firms in Urban Harbin. The main ideas were as follows: First of all, generalize the data of road and settlement into a basic geographic layer using cartographic generalization. Secondly, analyse the agglomeration effect of producer service firms using the local Getis-Ord Gi* statistic, and abstract into an enterprise thematic layer. Then overlay basic geographic layer with enterprise thematic layer to get the spatial distribution pattern of producer service firms.

\subsection{Analysis of the procedures}

The strategy to analyse the spatial distribution pattern of producer service firms includes seven steps. The details are as follows.

1. Generalize a basic geographic layer. Firstly, rank the roads according to the grade in the road attribute data, and extract road data with the high level to save as a main road layer. Secondly, abstract the main settlements, which have large area such as town centre, merge medium settlements, and save as a main settlement layer. Finally, overlay the main settlement layer to the main road layer to get the basic geographic layer.

2. Geocode enterprise data. According to the address of producer-service data, get the latitude and longitude of each enterprise. A point needs to note in this context. In order to ensure the geocoding accuracy, results should be checked by spatial topology relation. The inaccurate data need to be rematched artificially.

3. Convert the type of enterprise data from table into shape. The enterprise data saved in the table should be converted into shape file. Moreover, define the geographic coordinates system and transform into project coordinates system.

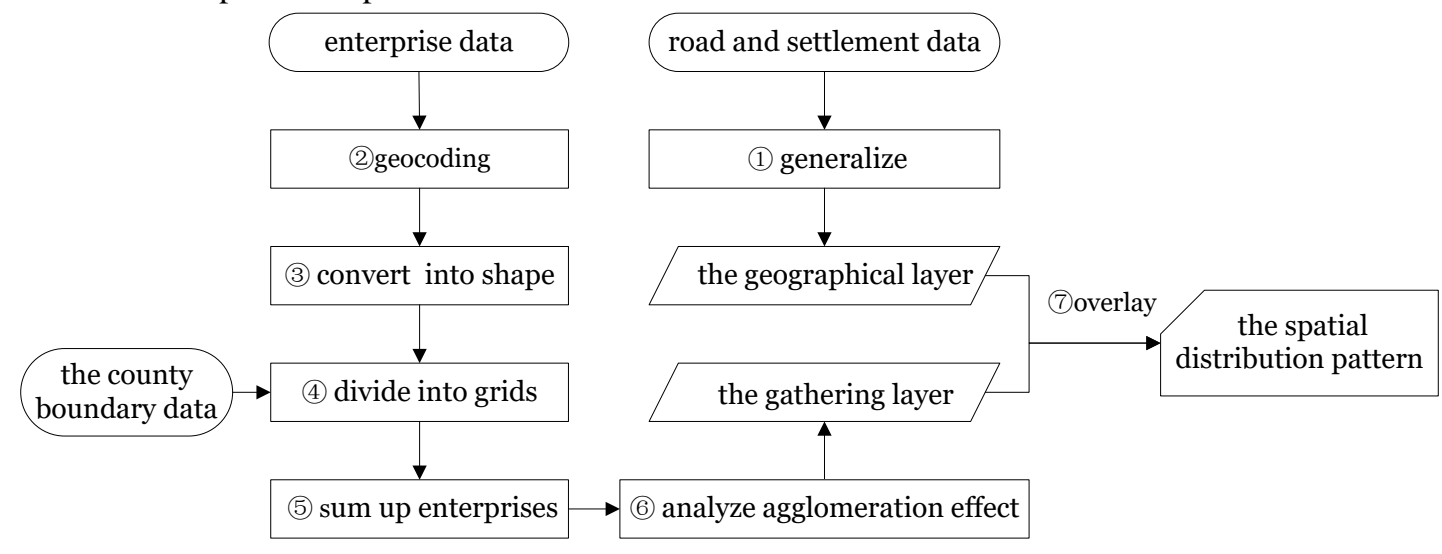

Figure 1 The chart of research process

4. Divide the study area into grids. Divide the study area into $1 \mathrm{~km} * 1 \mathrm{~km}$ grids in the county boundary layer. Specifically, get the minimum circumscribed rectangle of the county boundary, draw grid from left to right and from top to bottom in order. If the remaining region is less than $1 \mathrm{~km}^{2}$, draw a $1 \mathrm{~km} * 1 \mathrm{~km}$ grid beyond the scope.

5. Statistic the number of enterprises in each grid. Statistic the number of producer service firms in each grid, and save the results into the attribute table of the grid layer.

6. Analyse the agglomeration effect. Analyse the agglomeration effect of producer service firms by using the local Getis-Ord Gi* statistics. And the result will be put out as the enterprise thematic layer.

7. Summarize the spatial distribution pattern. Convert the enterprise thematic layer from grid type into shape type. Then overlay the enterprise thematic layer into the enterprise thematic layer and put out the result. Summarize the spatial distribution pattern of producer service firms with the output layer.

\subsection{Geocoding method}

Geocoding is often used to transform a physical address description to a location on the Earth's surface with geographic information systems (GIS) (Ward, M. H., et al. 2005). It uses a given address to identify geographic location on a given map ( Yi-feng Z., Jian-ping W., \& Yi C. 2007, Cun-qun S., Shun-ping Z., \& Lin Y. 2010). This paper uses a fuzzy Chinese-geocoding method based on the address elements identification mechanism to match enterprise data. It is essential that to find the closest matching address from the given address with the geographical coordinates. In fact, it is hard to find the same address in the given address. In order to improve the matching probability, a similar address is allowed within a specified tolerance range of error. Matching 
indicators (MI) is used to calculate the similarity between the matching address and the given address with the geographical coordinates. The formula of MI is given as follow.

$$
M I=\left(\begin{array}{lllll}
1,1, & \cdots, 1
\end{array}\right)\left(\begin{array}{cccc}
\frac{A_{1}}{U_{1}} Q_{11} & \frac{A_{2}}{U_{1}} Q_{12} & \cdots & \frac{A_{n}}{U_{1}} Q_{1 n} \\
\frac{A_{1}}{U_{2}} Q_{21} & \frac{A_{2}}{U_{2}} Q_{22} & \cdots & \frac{A_{n}}{U_{2}} Q_{2 n} \\
\vdots & \vdots & \ddots & \vdots \\
\frac{A_{1}}{U_{m}} Q_{m 1} & \frac{A_{2}}{U_{m}} Q_{m 2} & \cdots & \frac{A_{n}}{U_{m}} Q_{m n}
\end{array}\right)\left(\begin{array}{c}
1 \\
1 \\
\cdots \\
1
\end{array}\right)
$$

Where, $M I$ is the matching indicator between two addresses. $A_{i}$ stands for the $i$ th element in the matching address without coordinate. $U_{i}$ stands for the $j$ th element in the given address with the coordinates. And $Q_{i j}$ stands for the weight of element $A_{i}$. General, the value of weight depend on the importance of the element in the address. For example, the street has the higher value than the house number.

\subsection{Getis-Ord Gi*}

Spatial autocorrelation statistics analyse the degree of dependency among observations of a given geographic space. There are two type methods of spatial autocorrelation, global spatial autocorrelation and local spatial autocorrelation. The former describes the overall situation of a phenomenon, and the latter calculates the scope of aggregation. Local Getis-Ord Gi*, one of the local spatial autocorrelation, was used to analyse the agglomeration effect of producer service firms in this paper.( Anselin L. 1995, Robert H. 2009, Xiao-dong M., Quan-lin L. \& Yi S. 2012, Kaizhou Z. \& Nan C. 2014).

It need to calculate the Getis-Ord Gi* value of each grid. If a grid has a high value and be surrounded by other grids with high values as well, it will be called as a statistically significant hot spot. The local sum of a grid and its neighbours is compared proportionally to the sum of all features. When the local sum is very different from the expected local sum, and the difference is too large to be the result of random chance, a statistically significant score results will be gotten. The formula is as follows.

$$
G=\frac{\sum_{j=1}^{n} w_{i j} x_{j}-X \sum_{j=1}^{n} w_{i j}}{S \sqrt{\frac{n \sum_{j=1}^{n} w_{i j}^{2}-\left(\sum_{j=1}^{n} w_{i j}\right)^{2}}{n-1}}}
$$

Where $x_{j}$ is the attribute value for feature $\mathrm{j}, w_{i j}$ is the spatial weight between feature $i$ and $j, n$ is equal to the total number of feature and $X=\frac{\sum_{j=1}^{n} x_{j}}{n}$, $S=\sqrt{\frac{\sum_{j=1}^{n} x_{j}^{2}}{n}-X^{2}}$

\section{Study area and Testing Data}

\subsection{Study area}

As the capital of Heilongjiang Province, Harbin is the centre of political, economic and cultural in north-eastern China. Like many other metropolitans in China, Harbin has been experiencing rapid urbanization and motorization after the reform and opening-up in 1978. By the year 2016, Harbin covers an area of 10198 square kilometres with a total population of 9620500 . Urban Harbin contains 8 districts with $18.94 \%$ of area, $47.45 \%$ of population of Harbin. Statistics show that 41,482 producer service enterprises are located in Urban Harbin, making up $83.36 \%$ of the total number of Harbin.

\subsection{Testing Data}

The experimental data including geographical data and producer service firms data. The former, provided in vector format by China Cartographic Publishing House, contains the road layer, the settlement layer and the county boundary layer of Urban Harbin in 2016. The latter, provided in a tabular format by the National Administration for Code Allocation to Organization, with

\begin{tabular}{|c|c|c|c|c|c|c|c|c|}
\hline & Daoli & Nangang & DaoWai & Pingfang & Songbei & Xiangfang & Hulan & Acheng \\
\hline Financial and insurance industry & 524 & 777 & 240 & 47 & 34 & 283 & 80 & 110 \\
\hline Real estate industry & 1208 & 1488 & 643 & 95 & 176 & 769 & 159 & 86 \\
\hline Commercial service industry & 3082 & 5649 & 1285 & 174 & 221 & 2464 & 79 & 100 \\
\hline $\begin{array}{l}\text { Information transmission, software and information } \\
\text { technology service industry }\end{array}$ & 473 & 2205 & 324 & 87 & 74 & 498 & 10 & 13 \\
\hline scientific research and technical service industry & 1391 & 3945 & 961 & 83 & 161 & 1352 & 55 & 55 \\
\hline Transport, storage and postal service industry & 593 & 917 & 1222 & 245 & 44 & 1010 & 93 & 369 \\
\hline Education culture, radio and television industry & 1181 & 2157 & 578 & 139 & 88 & 1132 & 133 & 121 \\
\hline Total & 8452 & 17138 & 5253 & 870 & 798 & 7508 & 609 & 854 \\
\hline
\end{tabular}

Table 1. Statistics of producer service firms in each district of Urban Harbin. 
with some required fields of producer service firms such as the name, the address, the location, the organization code, the type of national economy industry, the registered capital, etc. This paper build analysis and interpretation on the foundations provided by literatures documenting the internationalization of producer service firms including financial and insurance industry, real estate industry, commercial service industry, information transmission, software and information technology service industry, scientific research and technical service industry, transport, storage and postal service industry, and education culture, radio and television industry. According to the national economic industry classification (2011) and the administrative division code, a total of 41,482 pieces of producer service firms in 8 districts of Urban Harbin were obtained. The statistics of producer service firms data are shown in Table 1. This table lists the number of each type of national economy industry in 8 districts of Urban Harbin.

\section{Experimental results}

This paper, using the proposed strategy, analysed the spatial distribution pattern of producer service firms in urban Harbin. More details, including geocoding, generalize the basic geographic layer, analyse the agglomeration effect, and get the spatial distribution pattern of producer service firms, are shown as follows.

\subsection{Geocoding and evaluation}

This paper matched each data of producer service firms data using Chinese geocoding method. Result shows that 39997 pieces of data were matched automatic. In order to evaluate the accuracy, 2000 pieces of data were chosen randomly and gotten the geographical coordinates artificially. The positional error of each piece data, which measured by the distance between the automatic coordinate and artificial coordinate were calculated to evaluate the accuracy. Result shown the maximum positional error was $2314.5 \mathrm{~m}$ and the average number was $132.74 \mathrm{~m}$. The counts of the positional error within $50 \mathrm{~m}, 100 \mathrm{~m}, 500 \mathrm{~m}, 1000 \mathrm{~m}$ and more than $1000 \mathrm{~m}$ were calculated respectively. The cumulative graph of positional error are shown in Figure 2. It is obviously that more than $65.9 \%$ of data were matched the geographical coordinates accurately. And $98.7 \%$ of data were matched within the $1000 \mathrm{~m}$ positional error. As the analysis based on the $1 \mathrm{~km}^{2}$ grid, $1000 \mathrm{~m}$ positional error was acceptable.

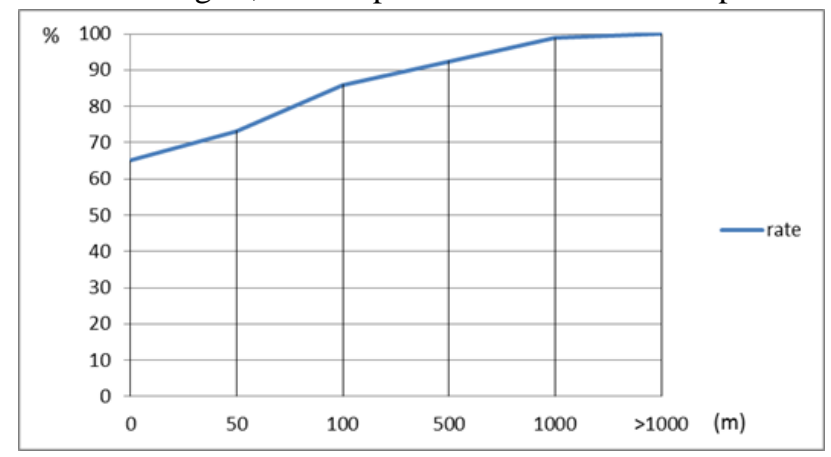

Figure 2 the cumulative graph of positional error.

\subsection{Geographical generalization}

According to the road grade and the settlements, Urban Harbin including six regions: the centre region, the transition region, the surrounding areas, the peripheral district, the peripheral district centre and peripheral villages. Specifically, there are three circle areas $(126.65$ $\left.\mathrm{E}, 45.75^{\circ} \mathrm{N}\right)$ with the radius of about $5 \mathrm{~km}, 8 \mathrm{~km}$ and 15 $\mathrm{km}$ in the centre of Urban Harbin. In particular, the centre region, including Daoli district, Daowai district, most of Nangang district and a part of Xiangfang district, is located among the South Straight, Gongbin Road, Hexing Road and Xinda Street. The transition region, including urban Xiangfang district and urban Songbei district is among Xilong Street, Qianweida Street, Xinshan Road and Zhongyuan Street. The surrounding areas is inside of high-speed beltway of Urban Harbin. What's more, Hulan district, Pingfang district and ACheng district are located to the North, South and southeast of the surrounding areas. Both the peripheral district and the peripheral district center are summarized to express the above region. In addition, there are some peripheral villages outside of the the surrounding areas. The basic geographic layer of urban Harbin is shown in Figure 3.

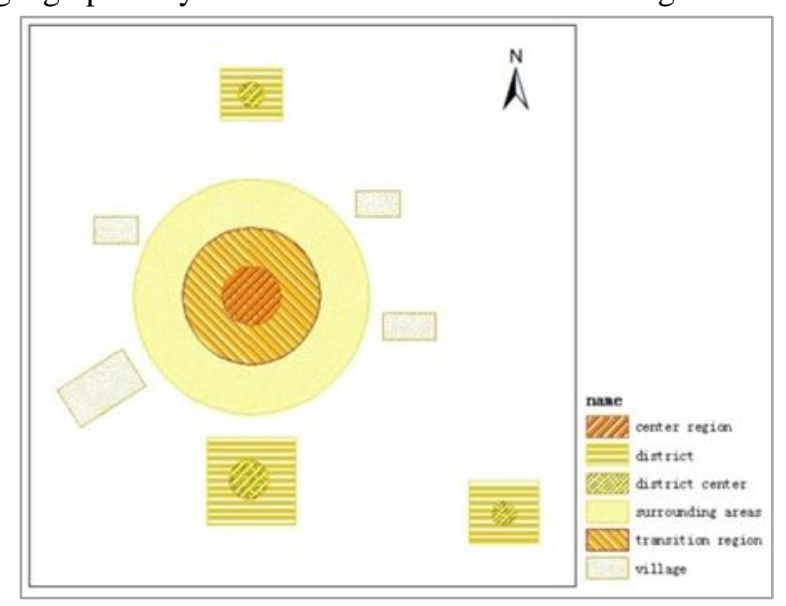

Figure 3 The basic geographic layer of urban Harbin

\subsection{Analysis on agglomeration effect}

This paper, using local Getis-Ord Gi* statistic to analyse the agglomeration effect of producer service firms of Urban Harbin. The result shows that there are 5 categories of agglomeration effect in Urban Harbin, including high heat zone, heat zone, random area, cold zone and high cold zone. The enterprise thematic layer is shown in figure 4 . Three of the categories contained the high heat zone, the heat zone and the cold zone are needed.

The high heat zone is located in the centre of Urban Harbin, covering the Nangang, Xiangfang, Daowai and Daoli District, and among the area of Hexing Road, Jiangpan Road and South Straight. Besides there are three regions appeared the high heat agglomeration effect obviously, including south of Zhongyuan avenue in Songbei district, the area which is near the Xinjiang street in Pingfang district and the area between the Liberation avenue and Pailu Road in Acheng district. 


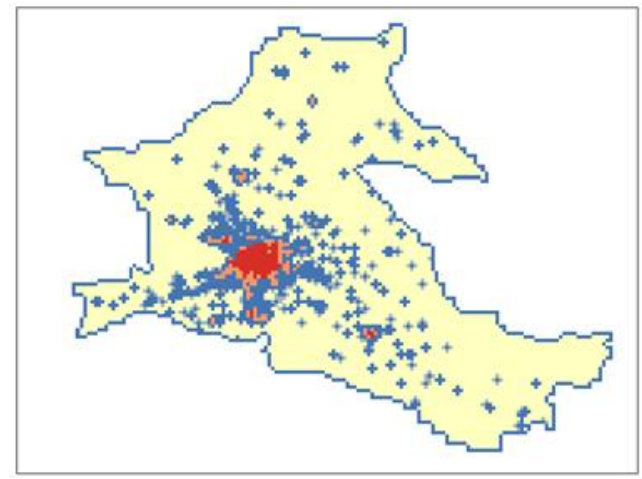

Figure 4 The agglomeration of producer service firms in Urban Harbin.

There are five regions of the heat zone outside of the high heat zone. The first region is located among Chengxiang Road, Xuefu Road, Yuexing Road, Tongxiao Street and Huagong Road. The second region is located among Zhongyuan avenue, Haping Road, Youxie Street and Nanguang Street. The third region is nearby Yanchuan Street in Acheng District. The forth region is nearby North and South Street in Hulan district. And the last region is dispersed in some area of HaSui Speedway, National Highway G102.

The cold zone is scattered in the Acheng district, Pingfang district and Hulan district, which widely located in the periphery of the heat zone. Most of them are in Songbei District, Hulan district and Pingfang district.

Generally, the agglomeration effect of the producer service firms in urban Harbin is obvious. A portion of central urban Harbin appears high heat agglomeration effect, including the center city of Pingfang district, Songbei and Acheng district. The region surrounding the high heat zone appears the heat agglomeration effect. Besides someplace, nearby the highway or national highway also shows the heat agglomeration effect. Most area within the Acheng district, Pingfang district and Hulan district appears cold agglomeration effect. The zone in Songbei, Hulan and Pingfang district appears apparently high cold agglomeration effect.

\subsection{Spatial distribution pattern}

This paper surmised the spatial distribution pattern of the producer service firms in Urban Harbin on the basis of overlaying the basic geographic layer and the enterprise thematic layer. The spatial distribution pattern of producer service firms in Urban Harbin is shown in figure 5.

The results indicated four distribution regions: (i) it showed the high hot agglomeration effect in centre urban Harbin; (ii) it appeared hot agglomeration effect in both north and south of centre urban Harbin; (iii) some of producer service firms randomly distributed in the surrounding area; and (iv) a fraction of producer service firms scattered in the peripheral area. In particular the result shows that the spatial distribution pattern of producer service firms in three circle areas has evident agglomeration effect. The inner area is the highest gathered area, the middle area is higher gathered area, and the outer is high gathered area.

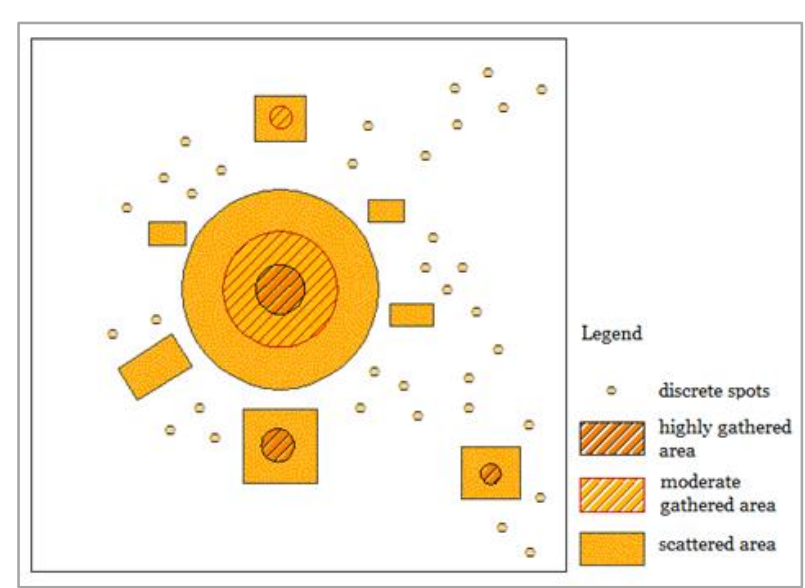

Figure 5 The spatial distribution pattern of producer service firms in Urban Harbin.

\section{Conclusions}

This paper proposed a strategy to analyse the spatial distribution pattern using cartographic generalization, the local Getis-Ord Gi* in ESDA and the overlay method of GIS. And surmise the spatial distribution pattern of the producer service firms in Urban Harbin. The agglomeration effect analysed by the local Getis-Ord Gi* statistics, is based on the number of the producer service firms in each gird. While the scale of the enterprise is not considered as the lack of information. If the condition allows, some attributions such as the turnover, the number of employees and the amount of tax should be considered to correct.

\section{Acknowledgements}

We acknowledge the National Administration for Code Allocation to Organization, which provides the producer service firms data for our experiment. We would also thank the Chinese Academy of Surveying and Mapping, which provides research facilities. This work was supported by the National Key Research and Development Program of China (2016YFC0803108) and the Chinese Academy of Surveying and Mapping Fundamental Research Fund (No.7771812)

\section{References}

Anselin L. (1995) Local indicators of spatial associationLISA. Geographical Analysis 27(2):93-115.

Bagchisen, S., \& Sen, J. (1997). The Current State of Knowledge in International Business in Producer Services. Environment and Planning A, 29(7), 1153-1174. Bathelt, H., Malmberg, A., \& Maskell, P. (2004). Clusters and Knowledge: Local Buzz, Global Pipelines and the Process of Knowledge Creation. Progress in Human Geography, 28(1), 31-56.

Cun-qun S., Shun-ping Z., \& Lin Y. (2010) Chinese Geocoding Based on Classification Database of Geographical Names. Jourmal of Computer Application. 30 (7):19531955. 
Faulconbridge, J. R. (2008). Managing the Transnational Law Firm: A Relational Analysis of Professional Systems, Embedded Actors, and Time-Space Sensitive Governance. Economic Geography, 84(2), 185-210.

Feng Z., \& Hui L. (2008) Spatial Distribution of Urban Producer Service: A Case Study of Nanjing. Word Regional Studies 17(1), 24-31.

Greenfield, H. I. (1966). Manpower and the Growth of Producer Services. Economic Development, 163.

Illeris S. (1996) The Service Economy: A Geographical Approach. Chichester: Wiley.

Jones, A. (2002). The "Global City" Misconceived: the Myth of "Global Management" in Transnational Service Firms. Geoforum, 33(3), 335-350.

Kaizhou Z. \& Nan C. (2014) Characteristics of Spatialtemporal Evolution in Population Aging and Driving Mechanism at County Level in Fujian Province during 1990-2010. Progress in Geography, 33(5), 605-615.

Marshall, J. N., \& Daniels, P. W. (1996). Service Industries in the World Economy. The Geographical Journal, 162(2).

Qian-hu C., Xin X., \& Hui-min S. (2008) Spatial Evolution of Producer Services in Hangzhou. City Planning Review 32(8), 48-52.

Qun-yi Z., \& Yi-xing Z. (2007). Spatial Structure of Producer Services in Beijing Metropolitan Area. City Planning Review, 31(5), 24-31.

Rober H. (2009) Spatial Data Analysis Theory and Practice. 190-203.

Shearmur R., \& Alvergne C. (2002) Intrametropolitan Patterns of High-order Business Service Location: A Comparative Study of Seventeen Sectors in Lie-deFrance. Urban Studies. 39(7),1143-1163.

Stanback (1991) The New Suburbanization: Challenge to the Central City. Boulder Co Western Press.

Taylor, P. J., Derudder, B., Faulconbridge, J. R., Hoyler, M., \& Ni, P. (2014). Advanced Producer Service Firms as Strategic Networks, Global Cities as Strategic Places. Economic Geography, 90(3), 267-291.

Wen-zhong Z. (1990) A Study of Metropolitan Service Industry Location Theory and Substantiation. Geographical Research 18(3), 273-281.

Ward, M. H., Nuckols, J. R., Giglierano, J., Bonner, M. R., Wolter, C., \& Airola, M., et al. (2005). Positional accuracy of two methods of geocoding. Epidemiology, 16(4), 542-547.

Xiao-dong M., Quan-lin L., \& Yi S. (2012) Morphological Difference and Regional Types of Rural Settlements in Jiangsu Province. Acta Geographica Sinica, 67(4), 516-525.

Yi-feng Z., Jian-ping W., \& Yi C. (2007) The Improvement of Geocoding in ArcGIS. Geomatics \& Spatial Information Technology, 30(3):116-118.

Yin C., \& Jin-yong L. (2004) Study on the Location Mode of Production Service Industry and Relative Institutions. Shanghai Economic Review (7), 52-57.
Yu-fang S., \& Shu-hua L. (2011) Study on the Layout Structure and Tendency of Producer Services of the Yangtze River Delta Area. Urban Studies 18(4), 57-64. 